\title{
Tobacco Use Increases Oxidative DNA Damage in Sperm - Possible Etiology of Childhood Cancer
}

\author{
Shiv Basant Kumar ${ }^{1}$, Bhavna Chawla ${ }^{2}$, Shilpa Bisht ${ }^{1}$, Raj Kumar Yadav ${ }^{3}$, Rima \\ Dada $^{1 *}$
}

\begin{abstract}
Background: Cigarette smoking and tobacco chewing are common modes of consuming tobacco all over the world. Parents need to be aware that germ cell integrity is vital for birth of healthy offspring as biological parenting begins much before birth of a child and even before conception. The present study was conducted to determine the etiology of non-familial sporadic heritable retinoblastoma (NFSHRb), by evaluating oxidative sperm DNA damage in fathers due to use of tobacco (smoking and chewing). Materials and Methods: We recruited 145 fathers of NFSHRb children and 53 fathers of healthy children (controls) in the study. Tobacco history was obtained by personal interview. Seminal reactive oxygen species (ROS) in semen, sperm DNA fragmentation index (DFI) and 8 hydroxy 2' deoxyguanosine (8-OHdG) levels in sperm were evaluated. The RB1 gene was screened in genomic blood DNA of parents of children with NFSHRb and controls. Odds ratios (ORs) derived from conditional logistic regression models. Results: There was significant difference in the levels of $\operatorname{ROS}(p<0.05)$, DFI $(p<0.05)$ and 8-OHdG $(p<0.05)$ between tobacco users and non-users. The OR of NFSHRb for smokers was 7.29 (95\% CI 2.9-34.5, $p<0.01)$, for tobacco chewers $4.75(2.07-10.9, p<0.05)$ and for both 9.11 (3.79-39.2; $p<0.01)$. Conclusions: This study emphasizes the adverse effect of tobacco on the paternal genome and how accumulation of oxidative damage in sperm DNA may contribute to the etiology of NFSHRb. In an ongoing parallel study in our laboratory, 11 of fathers who smoked underwent. Meditation and yoga interventions, showed significant decline in levels of highly mutagenic oxidised DNA adducts after 6 months. Thus our lifestyle and social habits impact sperm DNA integrity and simple interventions like yoga and meditation are therapeutic for oxidative damage to sperm DNA.
\end{abstract}

Keywords: Sperm DNA damage - childhood cancer - retinoblastoma - tobacco - smokers - oxidative stress

Asian Pac J Cancer Prev, 16 (16), 6967-6972

\section{Introduction}

In the recent years, there has been a decline in male reproductive health and standard semen parameters (sperm count, morphology and motility). We have also observed elevated levels of seminal free radicals and oxidative sperm DNA damage in normozoospermic men. The rapid decline in semen quality may be due to life style factors coupled with increasing exposure to environmental pollutants, endocrine disruptors, poor life style habits, smoking, alcohol intake and highly stressful life.

Over the past decade, the interest in assessing the impact of social and lifestyle habits on health has increased. Socioeconomic characteristics have been associated with morbidity and mortality discrepancies in many developed and undeveloped countries (Khan., 2005). Recent studies have documented that though the genome accumulates mutations but it is relatively stable as compared to the dynamic epigenome (Paula et al.,
2013). The sperm epigenome is highly sensitive to the external and internal environment thus highly sensitive to environmental pollution and our life style habits. Also, a number of environmental factors (e.g. toxicants) have been shown to promote the epigenetic transgenerational inheritance of disease and phenotypic variation. Environmentally induced epigenetic transgenerational inheritance of disease and phenotypic variation involves the germ line transmission of altered epigenetic information in the absence of direct exposure (Skinner et al., 2013). In recent years there are increasing number of studies emphasizing the deterioration in sperm quality and more couples adopting assisted conception technologies. There are reports that children conceived by ART are at slightly increased risk of childhood cancers, autosomal dominant disorders, and neuropsychological disorders (Aitken et al., 2014).

In a recent study Tabrizi et al. (2015), proposed that environmental exposures such as pre and post natal

${ }^{1}$ Laboratory for Molecular Reproduction and Genetics, Department of Anatomy, ${ }^{2}$ Ocular Oncology \& Pediatric Ophthalmology Service, Rajendra Prasad Centre for Ophthalmic Sciences, ${ }^{3}$ Integral Health Clinics, Department of Physiology, All India Institute of Medical Sciences, New Delhi, India*For correspondence: rima_dada@rediffmail.com 
exposure to high voltage power lines and pollutants can affect both parents and children (Tabrizi et al., 2015).

Oxidative stress is also known to up regulate levels of DNA methyltransferases (DNMT) and thus seminal oxidative stress may lead to epigenetic alterations and a recent study has reported that paternal sperm DNA methylation is associated with early signs of autism (Feinberg et al., 2015). Retinoblastoma represents almost $4 \%$ of all pediatric malignancies (Meel et al., 2012). Munlima Hazarika et al, reported that in Asian region $\mathrm{Rb}$ is most prevalent form of childhood cancer next to leukemia (Hazarika et al., 2014). Studies from India show a two- to three-fold higher incidence of tumors of the eye (majority of which are retinoblastoma) in children (Meel et al., 2012).

Cigarette smoke contains many well-established carcinogens; both active and passive smoking have been implicated in the development of several cancers during adulthood (Sobus et al., 2014). Cigarette smoke has been linked to an increased frequency of chromosomal abnormalities and aneuploidy in sperm (Pluth et al., 2000). The rate of adolescent addiction to cigarettes and tobacco use is high in India. According to Global Adult Tobacco Survey 2009, in India, more than half of the smokers begin smoking in their adolescence (GATS India report, 2014).

In the present study, we have used the sperm chromatin structure assay (SCSA), which measures DNA damage by flow cytometry by analyzing the susceptibility of sperm nuclear DNA to in situ denaturation followed by Acridine Orange staining (Evention and Wixon, 2006). The SCSA technique is now included in a variety of largescale epidemiological studies to detect more subtle male reproductive health effects of environmental toxicants and has already been deployed to study possible DDT induced sperm DNA damage (Evention and Wixon, 2005; Rignell et al., 2005). Alteration of sperm epigenome may cause hypermethylation of tumour suppressor genes and hypomethylation of repetitive elements may be the underlying pathology in oncogenesis (Rignell et al., 2005; Haoyang et al., 2013). Thus tobacco use in any forms not only harmful to the active user but also affects paternal genome which accumulates damage even before conception of child.

Therefore, the current study aimed to assess the effect of use of tobacco by father's in the etiology of oxidative sperm DNA damage which may lead to NFSHRb in the offspring. This information might indirectly add to our understanding of the biologic link between tobacco use and its effect on DNA integrity by evaluating seminal oxidative stress, sperm DNA damage and oxidative DNA damage by assaying 8-hydroxy-2'-deoxyguanosine (8$\mathrm{OHdG}$ ) levels. 11 fathers underwent yoga/meditation intervention. Findings of this study highlight that majority of sperm DNA damage is oxidative in nature as is indicated by raised levels of ROS and 8-OHdG. In this study we highlight the deterioration in sperm quality and accumulation of mutagenic bases which we believe may be the cause of childhood cancer and the significant decline in oxidative damage to Sperm genome following meditation and yoga intervention.

\section{Materials and Methods}

After approval from ethical committee of the institute (IESC/T-364), this study was planned on a cohort of fathers of children with NFSHRb at All India Institute of Medical Sciences, New Delhi, India. We recruited 145 fathers of children with NFSHRb for our study out of these $128(78 \%)$ children were diagnosed with unilateral and 17 (12\%) were bilateral Rb. Among them, 29 fathers declined to take part in the study; 26 were excluded due to mutations in $R B$ gene in fathers peripheral blood; 13 men were excluded as they previously had undergone vasectomy and varicocele. Thus, a total of 77 men (53\% of eligible subjects) were included. Control group consisted of 53 age-matched men with a healthy child born in the last 1 year. Only those cases were enrolled with no history of recent fever/infection/drug intake or change in life style last 1 year. Written informed consent was obtained from cases and controls before sampling.

Patients were then asked about their tobacco use pattern such as smoking habits specifically the number of cigarettes usually smoked per day over last 10 years before providing the sample and tobacco chewing habits in last 10 years (Niu et al., 2010). Also, detailed history was taken about social/occupational lifestyle habits of mothers. Any cases with intake of drug during pregnancy or mothers who smoked/consumed alcohol were excluded from this study. Both the parents were cytogenetically normal and did not harbor RB gene mutations.

In total, 11 fathers who smoked undertook daily practice of yoga and meditation in integral health clinic (IHC), AIIMS, New Delhi. All participants were attended 6 months program of meditation and yoga. Assessments are conducted at baseline (day 0), 3 and 6 months of follow-up. Measurement of conventional semen parameters was done in accordance to WHO, (1999) guideline. Various assays such as reactive oxygen species (ROS), DNA fragmentation index (DFI) and 8-hydroxy2'-deoxyguanosine (8-OHdG) which measures oxidative DNA damage were done.

\section{Semen collection and analysis}

Semen samples were obtained by masturbation in a sterile urine culture vial after 4 days of sexual abstinence. After liquefaction at $37^{\circ} \mathrm{C}$, semen analysis was performed as per the WHO guidelines (WHO laboratory manual., 1999).

\section{Estimation of Reactive Oxygen Species (ROS)}

After liquefaction, $400 \mu \mathrm{l}$ of neat semen was used to measure ROS levels. Ten micro litters of luminol (Sigma, USA), (5 mM) in dimethyl sulfoxide (DMSO), was added as a probe. A negative control was prepared by adding $10 \mu \mathrm{L}$ of $5 \mathrm{mM}$ luminol to $400 \mu \mathrm{L}$ of PBS. Levels of ROS were assessed by measuring the luminoldependant chemiluminescence with the luminometer (Sirius, Berthold Detection Systems GmbH, PFforzheim, Germany) in the integrated mode for 10 minutes. The results were expressed as $R L U /$ sec $/ 10^{6}$ sperm (Kumar et al., 2015). Each sample was analyzed in duplicate and the mean values were considered. 
Tobacco Use Increases Oxidative DNA Damage in Sperm - Possible Etiology of Childhood Cancer

Sperm chromatin structure assay (SCSA)

The SCSA was performed as per the protocol described earlier (Evention, 2013). Briefly, after complete analysis of the semen sample, the $\mathrm{X}$ - axis mean (red fluorescence) and Y- axis mean (green fluorescence) values were taken for sperm cells using Flow Jo software (Oregon, USA). Extent of DNA damage was expressed as DFI (Figure 1), which is the ratio of red to total (red and green) fluorescence, i.e., the level of denatured DNA over the total DNA (Evention et al.,2002). The percentage of high DNA stainability cells (HDS) were also recorded in each sample manually from the graph plot. HDS represents another distinct population in semen that characterizes immature spermatozoa with incomplete chromatin condensation. The DFI of the patients and controls were analyzed in duplicate, and the mean value was used for comparison.

\section{8-Hydroxy-2'-deoxyguanosine (8-OHdG) Assay}

8-OHdG was measured using ELİSA (Cayman Chemical, USA) as per manufacturer's protocol. The assay utilizes an anti-mouse IgG-coated plate and a tracer consisting of an 8-OHdG conjugate. This format has the advantage of providing low variability and increased sensitivity compared with assays that utilize an antigencoated plate. Procedure was as described in the protocol provided by the manufacturer. Absorbance was measured at $450 \mathrm{~nm}$ using an micro plate reader (Biotehnique,USA). The results were calculated with the Cayman data analysis system (Kumar et al., 2015).

\section{Mutation screening and sequence analysis}

Genomic DNA was extracted from whole blood using the organic method (Sambrook and Russell., 2006). Briefly, equal volume of RBC lysis buffer was added to the blood samples and centrifuged $\left(5000 \times \mathrm{g}, 4^{\circ} \mathrm{C}\right)$ after 10 minute incubation. To the cell pellet sodium dodecyl sulfate (SDS), DNA extraction buffer, Proteinase K was added and incubated overnight $\left(37^{\circ} \mathrm{C}\right)$. Phenol and chloroform: isoamylalcohol $(1: 1 \mathrm{~V} / \mathrm{V})$ was added to the suspension and mixed gently and centrifuged at $5000 \times$

Table 1. Comparison of ROS, DFI and 8-OHdG Levels between Fathers of NFSHRb Patient

\begin{tabular}{|c|c|c|c|}
\hline Parar & $(\mathrm{A}, \mathrm{n}=77)$ & $(\mathrm{B}, \mathrm{n}=53)$ & $\begin{array}{l}\text { P Value } \\
\text { (A) } V s(\mathrm{~B})\end{array}$ \\
\hline $\begin{array}{l}\text { ROS } \\
\text { (RLU/sec/million sperm) }\end{array}$ & & & $<0.05^{*}$ \\
\hline DFI $(\%)$ & 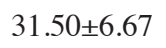 & $21.9 \pm 9.4$ & $<0.05^{*}$ \\
\hline 8-OHdG (ng/mL) & $63.02 \pm 2.91$ & $32.10 \pm 2.71$ & $<0.05^{*}$ \\
\hline
\end{tabular}

Cases (A) and Controls (B), *p value $<0.05$ was considered to be $\mathrm{g}$ for $10 \mathrm{~min}, 4^{\circ} \mathrm{C}$. Upper viscous layer was separated in different tube and using ice-cold ethanol DNA was precipitated. All 27 exons of RB1 including introns were amplified using genomic DNA trough polymerase chain reaction (PCR). Twenty seven sets of overlapping primers were designed with the help of the National Center for Biotechnology Information (NCBI) PRIMER3 program. PCR amplifications (S1000 thermal cycler, Bio-Rad, Hercules, CA) were done in a $25 \mu \mathrm{l}$ volume containing $2.0 \mathrm{pM}$ of each primer (Integrated DNA Technology, Coralville, IA), $50 \mathrm{ng}$ of genomic DNA, 1 unit of Taq polymerase (Banglore Genei PLtd, Bengaluru, Karnataka, India), $0.1 \mathrm{mM}$ of each deoxyribonucleotide triphosphate, and $2.0 \mu 1$ of $10 \times$ PCR buffer including $15 \mathrm{mM} \mathrm{MgCl} 2$, with 30 cycles of amplification, each consisting of $30 \mathrm{sec}$
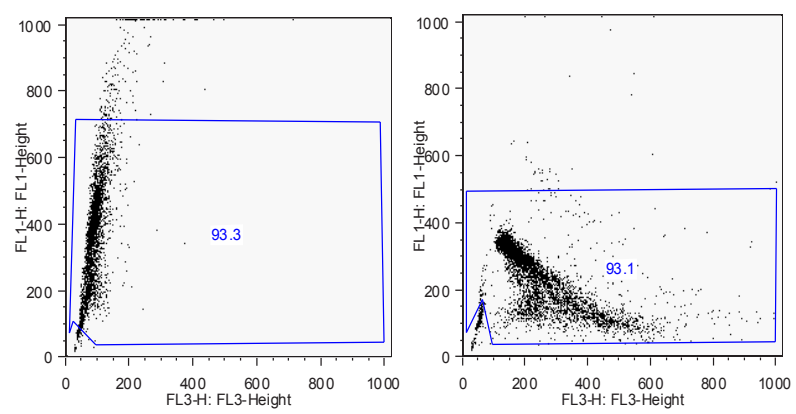

Figure 1. SCSA dot Plot Cytograms of Individual who did not use any Tobacco Products (A) and Individual who is smoker as well as tobacco chewer (B). X-axis( FL3) represents fragmented DNA and Y- axis (FL1) represents native DNA showing the percentage of spermatozoa with high levels of DNA fragmentation. Each dot represents a single spermatozoon

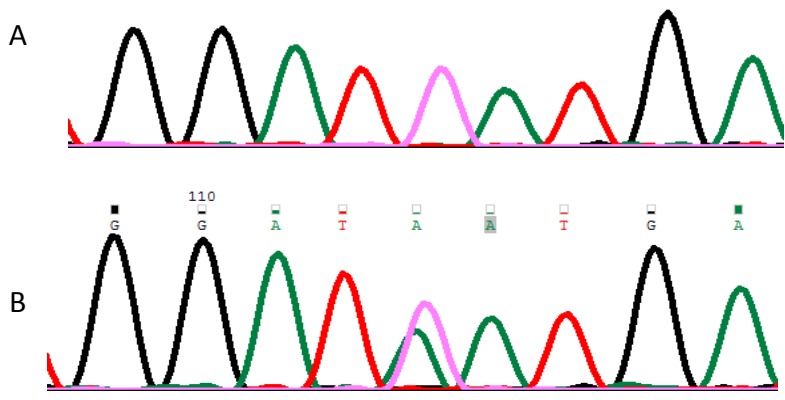

Figure 2. DNA Sequence from Exons 13 of RB1 gene. (A) The reference sequence derived from the control is shown. (B) The sequence derived from the fathers of NFSHRb patient. (Exon 13, C>A, g.64419,CAT>AAT, p.H339N)

Table 2. Comparison of ROS,DFI and $80 H d G$ levels between different groups

\begin{tabular}{lccccccc}
\hline Parameters $(\mathrm{n}=130)$ & $(\mathrm{A}, \mathrm{n}=33)$ & $(\mathrm{B}, \mathrm{n}=31)$ & $(\mathrm{C}, \mathrm{n}=41)$ & $(\mathrm{D}, \mathrm{n}=33)$ & $\begin{array}{c}\text { P Value } \\
(\mathrm{A}) V s(\mathrm{D})\end{array}$ & $\begin{array}{c}\text { P Value } \\
(\mathrm{B}) V s(\mathrm{D})\end{array}$ & $\begin{array}{c}\mathrm{P} \text { Value } \\
(\mathrm{C}) V s(\mathrm{D})\end{array}$ \\
\hline ROS & $36.0 \pm 1.83$ & $35.51 \pm 1.87$ & $43.11 \pm 7.23$ & $23.10 \pm 1.56$ & $<0.05^{*}$ & $<0.05^{*}$ & $<0.05^{*}$ \\
(RLU/sec/million sperm) & & & & & & & \\
DFI $(\%)$ & $31.50 \pm 6.67$ & $31.74 \pm 6.72$ & $39.57 \pm 3.91$ & $24.26 \pm 9.25$ & $>0.05$ & $>0.05$ & $<0.05^{*}$ \\
8-OHdG (ng/mL) & $66.02 \pm 2.91$ & $54.05 \pm 4.43$ & $178.59 \pm 19.78$ & $34.13 \pm 1.08$ & $<0.05^{*}$ & $<0.05^{*}$ & $<0.05^{*}$ \\
\hline
\end{tabular}

(A) Smokers; (B) Tobacco chewers; (C) Smokers + tobacco chewers; (D) Did not use any tobacco product at all, *p value $<0.05$ was Considered to be Significant 
Shiv Basant Kumar et al

denaturation at $95^{\circ} \mathrm{C}, 60 \mathrm{sec}$ annealing ranging from $58^{\circ} \mathrm{C}$ to $62^{\circ} \mathrm{C}$ and $1 \mathrm{~min}$ extension at $72^{\circ} \mathrm{C}$, and final extension at $72^{\circ} \mathrm{C}$ for $5 \mathrm{~min}$. Successfully amplified PCR products were purified using a PCR DNA fragments extraction kit (Geneaid Biotech Ltd.,Taiwan). Purified PCR products were sent for sequencing to MCLAB (Molecular Cloning Laboratories, South San Francisco, USA). DNA sequences were analyzed against the RB1 reference sequence (ENSG00000139687) using ClustalW2 provided by the European Molecular Biology Laboratory (EMBL) European Bioinformatics Institute (EBI).

\section{Statistical analysis}

Statistical analysis was performed using the SPSS 15.0 software (SPSS Inc., Chicago, IL, USA). Paired and unpaired corresponding differences in the life style parameters, SCSA, 8-OHdG and ROS values were examined using one-way repeated-measures analysis of variance (ANOVA). All the comparisons between controls and cases were calculated using Student's t-test. The twotailed $\mathrm{p}$ value $<0.05$ was considered as significant.

\section{Results}

The age of fathers of NFSHRb patient were $(33.17 \pm 11.2$ yrs) compared to $(31.5 \pm 4.54 \mathrm{yrs})$ in the controls. The standard semen parameters of fathers of NFSHRb patient were within the reference range were comparable (not significant) to that of controls. Among father of NFSHRb patient and controls we observed significant difference between experimental parameters such as ROS, DFI and 8-OHdG levels (Table 1).

In total (fathers of NFSHRb patient + controls, $\mathrm{n}=130$ ), there were $33(25 \%)$ smokers, 31 (23.84\%) tobacco chewers $41(31.53 \%)$ used both (tobacco chewers+ smokers) and 33 (25.38\%) did not use any form of tobacco at all. There was significant difference in all experimental parameters between tobacco users and no tobacco users (Table 2).

The odds ratio for smoker was [7.29(2.9-34.45; $\mathrm{p}<0.01)$; 95\%CI], tobacco chewers [4.75(2.07-10.91;

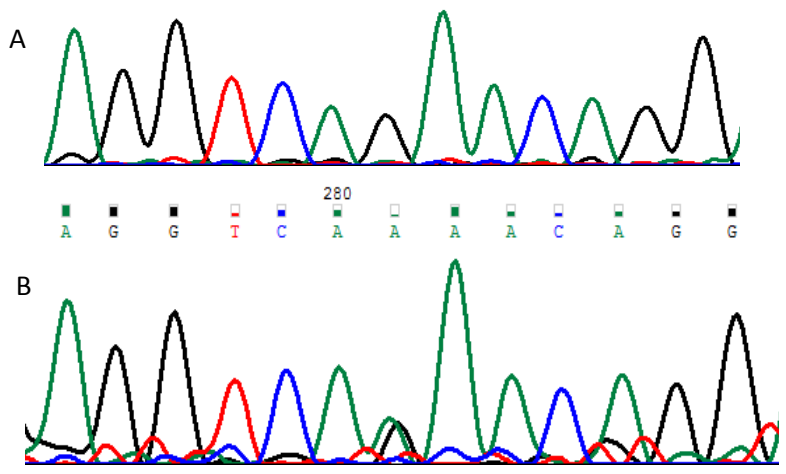

Figure 3. DNA Sequence from Intron 11-12 of RB1 gene. (A) The reference sequence derived from the control is shown. (B) The sequence derived from the fathers of NFSHRb patient. (Intron 11-12, G>A, c.771,CAG>CAA, p.Q257Q) (COSM923) $\mathrm{p}<0.05) ; 95 \% \mathrm{CI}$ ] and for both (smokers+ tobacco chewers) [9.11(3.79-39.17; $<<0.01)$; 95\% CI).

Twenty seven exons of the RB1 gene were screened in sperm DNA of 77 fathers of NFSHRb patient and 53 controls. There were two single nucleotide changes, one is $(\mathrm{C}>\mathrm{A})$ in exon 13 was observed in 14 fathers of NFSHRb patient (Figure 2. A and B) and another is $(\mathrm{G}>\mathrm{A})$ between exon 11 and 12 of the RB1 gene were observed in 12 fathers of NFSHRb patient (Figure 3. A and $\mathrm{B})$. These nucleotide changes resulted in replacement of cytosine (C) by adenine (A) at position g.64419 and replacement of guanine $(\mathrm{G})$ by adenine $(\mathrm{A})$ at position c.771 respectively. None of the controls showed the presence of this nucleotide change in the RB1 gene. These cases were excluded from the study as they had mutation in their somatic cells.

Meditation and yoga intervention: After 10 days of practice of meditation and yoga there was significant decline in free radical levels but there was no improvement in DNA integrity (DFI) and 8-OHdG levels. But after 6 months of meditation and yoga intervention all three parameters i.e ROS, DFI \% and 8OHdG levels showed significant decline.

\section{Discussion}

India is the third largest producer of tobacco and the second largest consumer of tobacco products worldwide (GATS India report, 2014). The Global Adult Tobacco Survey, India, 2009-2010 found that India was home to 274.9 million tobacco users, of which 163.7 million used only smokeless tobacco products such as tobacco lime, and betel quid with tobacco (GATS India report, 2014). About 4000 compounds are produced by single puff of cigarette through variety of processes including; hydrogenation, oxidation, decarboxylation and dehydration (Hammond et al., 2006). Many reports claim that the microelements present in smoke may penetrate the blood testis barrier to affect spermatogenesis, either by affecting genetic integrity or hormone production; our results also suggest the same (Toppari et al., 1996). So, not only consumption of tobacco affects the individual itself but also affects paternal genome even before conception of child.

Environmental factors (external and internal factors) like pollutants, global warming and exposure to environmental endocrine disruptors may affect integrity of sperm genome specially the sperm nuclear periphery where DNA is loosely bound to histones in the nuclohistone component (Jeng HA., 2014). Effects may be at different stages of the cell cycle such as during meiosis or spermiogenesis, and such abnormalities can have deleterious effects on offspring (Dinham and Malik, 2003). On the basis of our study, the evidence from tobacco use and sperm integrity consistent enough to elucidate the harmful effect of tobacco to sperm DNA quality.

If the sperm DNA damage not repaired then it causes persistence of oxidative DNA damage. As sperm has a highly limited cytosolic antioxidants and limited base exicision repair mechanisms by virtue of it being transcriptionally and translationally inert, the DNA damage borne in sperm DNA persists (Attia et al., 2014). 
A study from our lab documented that significantly lower levels of PARP1 and OGG1 in mature sperm of infertile men (Mishra et al.,2013; Thilagavathi et al., 2013). In addition, APE1 and XRCC1 are absent in sperm (Thilagavathi et al.,2013). Thus sperm depends entirely on oocyte to remove the oxidized bases. However, extensive sperm DNA damage and in certain cases suboptimal or aged oocytes are unable to clear entire damage. The cell then enters ' $S$ ' phase and damage is then transmitted to every cell of zygote making the embryo highly vulnerable to pre post implantation losses, congenital malformations and even childhood cancers (Kumar et al., 2012; Aitken et al., 2014). Also, about $90 \%$ of Rb cases occur due to germline mutation in the RB1 gene. A strong association between germline mutation and paternal age has been demonstrated by Leila Saremi et al (Saremi et al., 2014). This suggests a higher incidence of childhood cancer including retinoblastoma among children of older parents.

Simple life style interventions (meditation and yoga) caused a significant decline in both free radical levels but it took much longer (6 months) for reduction in sperm DNA damage (DFI\%) and 8OHdG levels. Thus adoption of this simple life style intervention may positively impact sperm DNA integrity and reduce disease burden in the next generation. Aitkin RJ, et al. documented that damage in sperm DNA may be the etiology of several neuropsychiatry disorders, autosomal dominant disorders, and even childhood cancers (Aitken et al., 2014).

We have also shown that practice of meditation and yoga not only causes a significant decrease in markers of inflammation but also rapid and significant decline in seminal free radical levels and a slower but significant improvement in DNA quality (decreased DFI) following 6 months practice of meditation and yoga.

It is the first time that such an association has emerged in a large-scale study, probably because, for the first time, sperm DNA damage due to use of tobacco has been correlated with ROS, DFI and 8-OHdG levels in fathers of children with non-familial sporadic heritable retinoblastoma.

As our data suggest that smoking causes increased free radical production which results in sperm DNA damage as seen by increased DFI. A two-step hypothesis has recently been put forward where defective spermatogenesis can lead to decreased sperm production and impaired chromatin remodeling with lower levels of nuclear protamination. In this condition, chromatin is more vulnerable and DNA becomes susceptible to various stressors, in particular oxidative stress (Aitken et al., 2014). Despite the certainty that androgen action is essential for the regulation of spermatogenesis in adult mammals, little is known about the mechanisms via which this regulation is exerted and by which smoking and its metabolites could affect these pathways. We can speculate that smoking and tobacco chewing; being estrogenic and antiandrogenic compounds could inhibit androgens from binding to their receptors, eventually leading to lower testosterone levels (Gray et al., 2001). However, binding to the specific receptors is only one of the several, and still unknown, ways a chemical can act as an endocrine disrupter (Tabb and Blumberg, 2006). The higher levels of oxidative DNA damage in sperm in smokers and tobacco chewers support the hypothesis that oxygen radicals are persistently produced in sperm because of use of tobacco in any form.

In conclusion, we used three different assays (ROS, DFI and 8OHdG) to evaluate the in vivo oxidative stress and genotoxicity due to use of tobacco, all of which showed that the tobacco causes significant levels of DNA damage in human sperm and the present study confirms that cigarette smoking adversely affects sperm DNA health. This study highlights that yoga and meditation are therapeutic for oxidative damage to sperm DNA. Understanding the mechanisms of DNA damage is critical for the analysis and management of tobacco-induced mutagenesis and carcinogenesis and its ultimate effect in various childhood cancer.

\section{Acknowledgements}

The authors would like to thank all participants including fathers of children with childhood cancer for providing with samples when ever required. This research was supported and funded by Indian council of Medical Research.

\section{References}

Aitken RJ, Smith TB, Jobling MS, et al (2014). Oxidative stress and male reproductive health. Asian J Androl, 16, 31-8.

Attia SM, Ahmad SF, Okash RM, et al (2014). Aroclor 1254-induced genotoxicity in male gonads through oxidatively damaged DNA and inhibition of DNA repair gene expression. Mutagenesis, 29, 379-84.

de Paula WB, Lucas CH, Agip AN, et al (2013). Energy, ageing, fidelity and sex: oocyte mitochondrial DNA as a protected genetic template. Philos Trans R Soc Lond B Biol Sci, 368, 20120263.

Dinham B and Malik S (2003). Pesticide and human rights. Into J Occupy Environ Health, 9, 40-52.

Evenson DP and Wixon R (2005). Environmental toxicants cause sperm DNA fragmentation as detected by the sperm chromatin structure assay (SCSA). Toxicol Appl Pharmacol, 207, 532-37.

Evenson DP and Wixon R (2006). Clinical aspects of sperm DNA fragmentation detection and male infertility. Theriogenol, 65, 979-91.

Feinberg JI, Bakulski KM, Jaffe AE, et al (2015). Paternal sperm DNA methylation associated with early signs of autism risk in an autism-enriched cohort. Int J Epidemiol, 0, 1-12.

GATS India report (2014). Ministry of Health and Family Welfare. URL: http://www.mohfw.nic.in/WriteReadData/ 1892s/1455618937GATS\%20 India.

Gray LE, Ostby J, Furr J, et al (2001). Effects of environmental antiandrogens on reproductive development in experimental animals. Human Reproduction Update, 2, 248-64.

Hammond D, Costello MJ, Fong GT, et al (2006). Exposure to tobacco marketing and support for tobacco control policies. Am J Health Behav, 30, 700-9.

Haoyang L, Xinzhou L, Yulin D, et al (2013). DNA methylation, a hand behind neurodegenerative diseases. Front Aging Neurosci, 5, 85 .

Hazarika M, Krishnatreya M, Bhuyan C, et al (2014). Overview of childhood cancers at a regional cancer centre in North-East India. Asian Pac J Cancer Prev, 15, 7817-9.

Jeng HA (2014). Exposure to endocrine disrupting chemicals and male reproductive health. Front Public Health, 2, 55. 
Shiv Basant Kumar et al

Khan MM (2005). Suicide prevention and developing countries. $J R$ Soc Med, 98, 459-3.

Kumar K, Thilagavathi J, Deka D, et al (2012). Unexplained early pregnancy loss:role of paternal DNA. Indian J Med Res, 136, 296-98.

Kumar SB, Chawla B, Seth R, et al (2014). Loss of paternal sperm DNA integrity in non- familial retinoblastoma. IOVS, $\mathbf{5 5}, 6440$.

Kumar SB, Gautam S, Tolahunase M, et al (2015). Improvement in sperm DNA quality following simple life style intervention: a study in fathers of children with non-familial sporadic heritable retinoblastoma. J Clin Case Rep, 5, 509.

Kumar SB, Yadav R, Yadav RK, et al (2015). Telomerase activity and cellular aging might be positively modified by a yoga-based lifestyle intervention. J Altern Complement Med, 21, 370-2.

Meel R, Radhakrishnan V, Bakhshi S (2012). Current therapy and recent advances in the management of retinoblastoma. Indian J Med Paediatr Onco, 33, 80-8.

Mishra SS, Kumar R, Malhotra N, et al.,(2013) Expression of PARP1 in primary infertility patientsandcorrelation with DNA fragmentation index a pilot study. J Anat Soc India, 62, 98-104.

Niu ZH, Liu JB, Shi TY, et al (2010). Impact of cigarette smoking on human sperm DNA integrity. Zhonghua Nan Ke Xue, 16, 300-4.

Pluth JM, Ramsey MJ, Tucker JD (2000). Role of maternal exposures and newborn genotypes on newborn chromosome aberration frequencies. Mutat Res, 465, 101-11.

Rignell-Hydbom A, Rylander L, Giwercman A, et al (2005). Exposure to PCBs and p, p'-DDE and human sperm chromatin integrity. Environ Health Perspect, 113, 175-9.

Sambrook J and Russell DW (2006). Isolation of Highmolecular-weight DNA from mammalian cells using proteinase $\mathrm{k}$ and phenol. CSH Protoc, 1, 2006

Saremi L, Imani S, Rostaminia M, et al (2014). Parental agerelated risk of retinoblastoma in Iranian children. Asian Pac J Cancer Prev, 15, 2847-50

Skinner MK, Guerrero-Bosagna C, Haque M, et al (2013). Environmentally induced transgenerational epigenetic reprogramming of primordial germ cells and the subsequent germ line. PLoS One, 8, 1-7.

Sobus SL and Warren GW (2014). The biologic effects of cigarette smoke on cancer cells. Cancer, 120, 3617- 26.

Tabb MM and Blumberg B (2006). New modes of action for endocrine-disrupting chemicals. Mol Endocrinol, 20, 47582.

Tabrizi MM and Bidgoli SA (2015). Increased risk of childhood acute lymphoblastic leukemia (ALL) by prenatal and postnatal exposure to high voltage power lines : a case control study in Isfahan, Iran. Asian Pac J Cancer Prev, 16, 2347-50

Thilagavathi J, Mishra SS, Kumar M, et al (2013). Analysis of telomere length in couples experiencing idiopathic recurrent pregnancy loss. J Assist Reprod Genet, 30, 793-98.

Toppari J, Larsen JC, Christiansen P, et al (1996). Male reproductive health and environmental xenoestrogens. Environ Health Perspect, 104, 741-803.

WHO laboratory manual (1999). Cambridge university press, cambridge. 